\section{MEN'S FEMININITY AND DOMINANCE ACROSS THE LIFESPAN: COHORT SIMILARITIES AND DIFFERENCES IN LIFESPAN TRAJECTORIES}

C. Jones ${ }^{1}$, D. Woods ${ }^{2}, 1$. California State University, 2. California State University, Fresno

Men low in femininity and high in dominance may be said to adhere to the traditional male gender role (Helson \& Soto, 2005). Developmental theory (Gutmann, 1994) and American sociocultural changes after 1970 (Strough et al., 2007) suggest all men will increase in FM and decrease in DO with age, but that younger cohorts will be more FM and less DO than older cohorts. Here we employ data from the Intergenerational Studies (IGS; Eichorn, 1981), focusing on original IGS men participants (born in the 1920s; $\mathrm{N}=147$ ) and sons of original participants (born in the 1950s; $\mathrm{N}=172$ ). The older cohort provides a maximum of 5 waves of data $(30 \mathrm{~s}$ through their 80 s); the younger cohort provides a maximum of 3 waves of data (20s through their 60s). We explore developmental trajectories of femininity (FM) and dominance (DO), assessed via the California Psychological Inventory (Gough \& Bradley, 1996), with a simultaneous focus on development, cohort, and family. Three-level longitudinal hierarchical linear modeling (HLM; Bryk \& Raudenbush, 1992) analyses indicate both younger and older cohort men remain stable and similar in level of FM across the lifespan. In contrast, the two cohorts significantly differ both in terms of DO intercept and slope. The older cohort enters young adulthood significantly more DO than the younger cohort and remains stable across the entire lifespan; the younger cohort enters young adulthood significantly less DO but significantly increases in DO across the entire lifespan. Results illustrate the complexities of development in the context of historical time.

\section{PREDICTING CHANGE IN PERCEIVED SOCIAL SUPPORT IN LATE LIFE: THE ROLE OF PERSONALITY AND GENDER}

\section{Jacobs ${ }^{1}$, R. Holtzer ${ }^{2}$, 1. Yeshiva University, 2. Albert}

Einstein College of Medicine

Perceived social support (PSS) has a uniquely beneficial effect on physical and mental health in older adults, yet relatively little is known about patterns of change in PSS during late life. While some individuals report rather stable PSS, others exhibit either increasing or decreasing levels. However, few studies have examined risk factors related to this individual-level change. Based on previous literature, we identified gender and personality (specifically neuroticism and extraversion) as primary variables of interest. Personality traits were analyzed independently and as combined NeuroticismExtraversion quadrants for the entire sample and in stratified models in order to explore the potential moderating effect of gender. The study sample consisted of 411 community-dwelling, healthy older adults $(56.2 \%$ female) who were followed for up to five years. Linear mixed effect models (LMEM) and Cox proportional hazards regression were used to examine continuous change and incidence of decline in PSS, respectively. LMEM results found that personality quadrants provided incremental information over single traits, with High Neuroticism-Low Extraversion and
Low Neuroticism-Low Extraversion significantly moderating PSS decline. Additionally, stratified models found High Neuroticism-Low Extraversion and High Neuroticism alone were linked with PSS decline in males, while Low Neuroticism-Low Extraversion was linked with PSS decline in females. LMEM and Cox models suggest that while PSS decline in males is likely associated with High Neuroticism, PSS decline in females may be associated instead with Low Extraversion. Future clinical implications include earlier identification of individuals who are at risk for decreased social support, and its related impact on health.

\section{SEXISM AND FRIENDSHIP BELIEFS AT MID-LIFE AND BEYOND}

S. Camardo ${ }^{1}$, T. Rieger ${ }^{2}$, C. Mehta ${ }^{2}$, 1. Emmanuel College, Boston, MA, USA, 2. Emmanuel College, 3. Emmanuel College/Children's Hospital Boston

The homosocial norm is the belief that friendships should occur exclusively between people of the same gender (Monsour, 2002: Rose, 1985). The homosocial norm posits that heterosexuality is viewed as normative and as such, cross-gender friendships are viewed with suspicion and disapproval (Savin-Williams, 2005). Homosocial tendencies have been associated with sexism in adolescence (Keener, Mehta, \& Strough, 2013), however little is known about the homosocial norm and sexism in older adulthood. In the current study we investigated associations between the homosocial norm and sexism in a sample of 203 (45 men) adults aged 50-92. Based on research with adolescents, we hypothesized that benevolent and hostile sexism would be positively associated with the homosocial norm for men, and negatively associated with the homosocial norm for women. Our hypothesis was partially supported. Specifically, we found that endorsement of the homosocial norm was positively associated with benevolent $(\mathrm{r}=.35, \mathrm{p}=.02)$ and hostile sexism $(\mathrm{r}=.39, \mathrm{p}=.009)$ for men. The homosocial norm was also positively associated with benevolent $(\mathrm{r}=.35, \mathrm{p}<.001)$ and hostile sexism $(\mathrm{r}=.31, \mathrm{p}<.001)$ for women. That the endorsement of the homosocial norm was associated with sexism for both older adult men and women may reflect a wider world view and beliefs about traditional roles and behaviors for men and women.

\section{SEXUAL PROBLEMS AND SEXUAL DISTRESS AMONG OLDER ADULTS: THE IMPORTANCE OF SEX MATTERS}

C. Juang ${ }^{1}$, B. Knight ${ }^{2}$, 1. Palo Alto VA Health Care System,

2. University of Southern Queensland

Sexual distress, or feeling bothered by sexual problems, is not always present when individuals have sexual problems. It is unclear who are more or less likely to be sexually distressed. Investigating individual differences in sexual distress allows us to further probe the mechanisms that would lead to higher sexual distress. The study examines whether sexual distress vary by gender, age group and partner status and explores whether one's attitude towards the importance of sex explain these differences. Sample included 1000 men and 1218 women who reported at least one sexual problem drawn from the National, Social Life, Health, and Aging 